\title{
A Malfunction Example of the Digital Wireless Communications between a Certain Type of Command Vehicle and Reconnaissance Vehicle
}

\author{
Xiaofang LOU, Huihua CHENG, Junxian LIU \& Hang XIAO \\ Wuhan Mechanical Technology College, Wuhan, China
}

\begin{abstract}
Digital wireless communications between a certain type of command vehicle and reconnaissance vehicle is an important communication way, it can solve the problems of communication distance and communication efficiency. Therefore, in the long distance communication, this way of communication is the first choice for us. In this paper, a malfunction which is encountered in the process of such communication mode is analyzed and excluded, the analysis result shows that in the process of such communication mode, the cable which is often pulled out and stuck in is often broken down, is the key point for us to exclude the malfunction.
\end{abstract}

KEYWORD: Command Vehicle; Reconnaissance Vehicle; Digital Wireless; Communication; Malfunction

Digital wireless communications between a certain type of command vehicle and reconnaissance vehicle is an important communication way in such equipment, which includes digital cable mode, digital wireless mode and network communication, etc. It is known that digital cable and network communication have to be carried out by a covered wire connection in the process of communication, so it is perhaps feasible if two vehicles are close enough in relative static condition. However, it is impractical to pull and construct so long a covered wire for communication in real combat when the two vehicles maintains a far distance from each other, because if length of the wire is too long, the communication would break off once malfunction occurred in a whatever local part of wire. Moreover, it is very difficult for troubleshooting. At this time digital wireless communication is vital for battlefield communication between the two vehicles, and as long as we well define the default parameters before combat, then you can communicate directly during combat. Undoubtedly, the digital wireless communication has become the most important means of communication between such equipment. So we here provide an example of malfunction diagnosis and troubleshooting in such type of digital wireless communication equipment for basic technical officers.[1]

\section{MALFUNCTIONPHENOMENON}

Open the command vehicle and the reconnaissance vehicle, operate a digital wireless communications network operation from initial step to final step to ensure that the process is definitely correct, and then get through the digital transmission and voice communication, but it comes out to find that both of communication ways fail and do not work at all.

\section{MALFUNCTIONDIAGNOSIS}

The main connection structure for wireless communication between command vehicle and reconnaissance vehicle is shown in Fig.1.

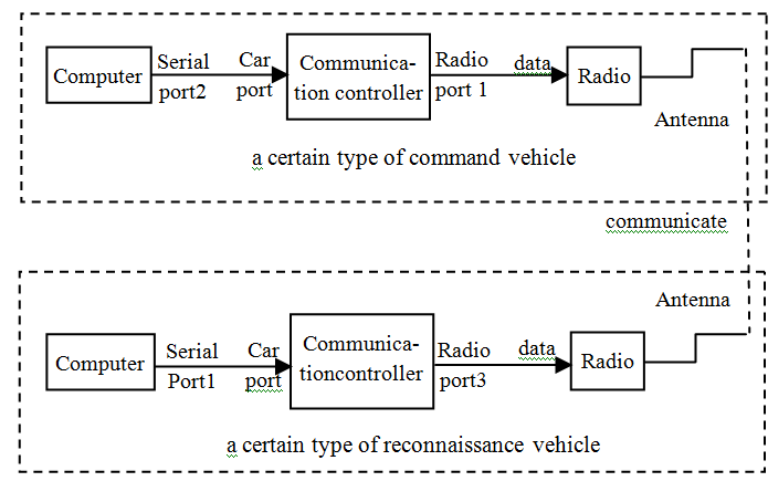

Fig. 1 schematic graph for connection between a certain type of command vehicle and reconnaissance vehicle 
As can be seen from Fig.1, the physical connection among the various devices is clear in the course of the entire wireless communications. In the command vehicle, the computer in the command caris connected to the car port of communication controller through its serial port 2, the communication controller is connected to the radio station through data port 1 and data port of radio station, and then the radio is connected to the counterpart of the respondent vehicle by each antenna on vehicles. In the reconnaissance vehicle, the connection among the computer, communication controller and command vehicles, is almost same as connection in the command vehicle. The slight difference is communication controller works on radio port 3 and the computer works by the serial port 1.Although the hardware connection has been done, in order to realize the communication between the various devices, it still requires software settings so as to carry out a data and words communication. So when we perform failure analysis and troubleshooting, solution should be carried out in two aspects, hardware and software. The detail is described as follows.[2]

\subsection{Software}

(1) Computer: IP address setting, incorrect setting of data delivery code.

(2) Communication controller: Wireless relay, static routing (set the destination node number to number of communication control unit of the respondent through their own radio ports), set time interval in radio management (it includes that wiring station number is set to be consistent with the local radio wiring, communication modes of both sides is set to be consistent, communication rate of two sides is identical, and two sides is set to the mode of master-slave.).

(3)Radio station: master-slave mode, one master and one slave, where other parameters ought to be consistent.

\subsection{Hardware}

(1) Computer: error in serial ports.

(2) Communication controller: radio board, interface board for data delivery, PX board (packet switching processing unit), LPU board (circuit switching unit), MPU board (main processing unit), TIME board (clock board), the user boards and other failures.

(3) Radio station: antenna, car adapter, host computer, cable fault.

\section{TROUBLESHOOTING}

\subsection{Software}

Firstly, check radio network settings of both sides. If there is no problem, then view the settings of both communication controllers secondly. If there is no problem, check the settings of both computers finally. If there is still no problem, the fault is estimated to not lie in the software settings, but in the hardware connection, and then failure elimination is focused on the hardware checking.[3]

\subsection{Hardware}

\subsubsection{Check the connection between the computer and the communication controller}

Firstly operate software of computer command and control system, reset the system management, and run the software for communications services after setting. If it is displayed that the computer registered number for data delivery is successful, then it proves that there is no error between the computer and the communication controller.

\subsubsection{Check the connection between the communication controller the radio station}

Run communication controller and check the radio status. If the radio status is found to be displayed correctly, then it indicates connection between the communication controller and the radio station is normal, and no failures message appears during the process of sending and receiving data and voice information.

\subsubsection{Check connection between the two radio stations}

(1) If communication is normal when plain words are transmitted, then it indicates that the antenna has no problem, neither radio station.

(2) Conduct frequency modulation (FM) calls, if it is found that the command vehicle is able to send command to the reconnaissance vehicle, but the reconnaissance vehicle can't resend message to the command vehicle. In the next, check state of the radio station in communication control of reconnaissance vehicle, if the words "unable to send" appear and the other parts are normal, then the communication controller malfunctions. If the replacement of good communication controller still does not work, then the radio station becomes the suspect of abnormal source. Perform a good test using the same model radio station, if fault persists, then fault does not come from the radio station itself under an initial determination. So this probably is a problem with the connection of the radio wiring. If replacement of a radio wire solves the problem, then the problem of the radio connection is confirmed. 


\section{REFERENCES}

[1] Wei Zhang \& Chuanfei Qiu \& Junxian Liu \& Quanzhi Zhou \& Zhichao Shao. 2010. Design and Implementation of DBFTesting System Based on FPGA. FCCC 2010:202205.

[2] Wei Zhang \& Zhichao Shao \& Huihua Cheng \& Quanzhi Zhou \& Xiaofang Lou \& Hang Xiao. 2012. Design and Realization of a Certain Type of Radio Power Amplify
Circuit Board Detecting and Teaching Platform. Information Technology for Manufacturing Systems III:800-804.

[3] Chuanfei Qiu \& Wei Zhang \& Wulei Tang \& Junxian Liu $\&$ Xiaofang Lou. 2012. The Design and Implementation of a Certain Communications Gateway with SOPC. ICDMA 2012:895-898. 